\title{
Tumor-derived exosomes educate fibroblasts to promote salivary adenoid cystic carcinoma metastasis via NGF-NTRK1 pathway
}

\author{
ZHENGLI XU $^{1,2^{*}}$, XING ZHENG $^{3 *}$ and JIANMING ZHENG ${ }^{2}$ \\ Departments of ${ }^{1}$ Stomatology and ${ }^{2}$ Pathology, Changhai Hospital, Second Military Medical University, Shanghai 200433; \\ ${ }^{3}$ Department of Stomatology, The Third Affiliated Hospital of Naval Medical University, Shanghai 200438, P.R. China
}

Received January 18, 2019; Accepted July 3, 2019

DOI: 10.3892/ol.2019.10740

\begin{abstract}
Salivary adenoid cystic carcinoma (SACC) is a rare head and neck malignancy characterized by unpredictable expansion, considerable perineural invasion and high risk of metastasis; however, the underlying mechanism of SACC progression remains unclear. Cancer-associated fibroblasts localized within the tumor microenvironment may promote cancer malignant transformation by enhancing tumor growth, blood vessel formation, inflammation development and metastasis occurrence. Small extracellular vesicles, including exosomes, are mediators of intercellular communication and can influence major tumor-associated pathways. The present study aimed to explore the exosome-mediated communication between SACC cells and fibroblasts. The results from confocal microscopy demonstrated that exosomes derived from the human cell line SACC- 83 were internalized by the human periodontal ligament fibroblast (HPLF) cells. Following exosome internalization, HPLF cells appeared to enhance SACC-83 cell metastasis and were educated toward a protumorigenesis phenotype according to transcriptome RNA sequencing and reverse transcription-quantitative polymerase chain reaction analysis. This phenomenon included exosome-mediated stimulation of proinflammatory cytokines and nerve growth factor (NGF) secretion. Furthermore, NGF blockage reduced the enhanced SACC-83 cell invasion stimulated by the supernatant isolated from exosome-educated HPLF cells. In addition, the results reported that neurotrophic receptor tyrosine kinase 1 (NTRK1), which is the high-affinity NGF receptor, was significantly upregulated in human SACC-83 cells. These results demonstrated that SACC-83
\end{abstract}

Correspondence to: Professor Jianming Zheng, Department of Pathology, Changhai Hospital, Second Military Medical University, 168 Changhai Road, Shanghai 200433, P.R. China

E-mail: jmzheng1962@163.com

*Contributed equally

Key words: salivary adenoid cystic carcinoma, exosome, fibroblast, metastasis, nerve growth factor, neurotrophic receptor tyrosine kinase 1 cell-derived exosomes educated HPLF cells toward the protumorigenic phenotype via the NGF-NTRK1 pathway, which suggested that this type of exosomes may be used as a potential therapeutic target for SACC.

\section{Introduction}

Salivary adenoid cystic carcinoma (SACC) is a rare head and neck malignancy, which has an incidence of 0.5-2.5 per 100,000 people with a slightly higher occurrence in women $(60 \%)$ in the USA $(1,2)$. The mean diagnostic age is $\sim 57$ years old and the overall 5-, 10- and 15-year survival rates are $\sim 90,80$ and $69 \%$, respectively (2). A total of $70-80 \%$ of SACC cases arises from the parotid glands, including the submandibular gland (10\%) or the sublingual and minor salivary glands (5\%) (1). Salivary gland malignancies are characterized by unpredictable growth expansion, considerable perineural invasion (PNI) and high risk of metastasis, which eventually leads to low survival outcomes (3). The lung is the most common metastatic site, followed by bone, liver and other sites, which account for 80, 15 and 5\% of SACC cases, respectively. Because of its low frequency and unique clinical characteristics, the underlying mechanisms of SACC metastasis and PNI remain unknown.

In addition to the extracellular matrix, cancer cells are surrounded by fibroblasts, vascular endothelial cells and immune cells (4). It has been demonstrated that cancer-associated fibroblasts (CAFs) localized within the tumor microenvironment can promote cancer malignant transition by enhancing tumor growth, blood vessel formation, inflammation and metastasis (5). CAFs exert pro-tumorigenic effects and provide a distinctive tumor microenvironment to promote tumor cell proliferation and invasion (6). The crosstalk between cancer cells and CAFs is critical for tumor initiation and progression, and targeting CAFs may therefore represent a promising therapeutic tool in the management of cancer (7).

Exosomes are cell-derived vesicles with a diameter of 40-100 nm, which comprise a variety of DNA, RNA, lipids and proteins that can be transferred from one cell to another via membrane vesicle trafficking (8). Previous studies demonstrated that intercellular communication between tumor-derived exosomes and all types of cell within the tumor microenvironment can influence cancer signaling pathways involving epithelial-mesenchymal transition, cancer growth, angiogenesis 
and metastasis $(9,10)$. For example, gastric cancer cells regulate liver microenvironment through epidermal growth factor receptor-containing exosomes, which can therefore lead to liver metastasis (9). Furthermore, bladder cancer cells can trigger fibroblast differentiation into CAFs through exosome-mediated transforming growth factor $\beta$ delivery and SMAD signaling activation (10). The role of exosomes in the crosstalk between SACC cells and CAFs requires therefore more attention.

The present study explored the mechanism underlying metastasis and PNI features in SACC mainly by focusing on the exosome-mediated communication between SACC cells and CAFs. The downstream effect of this communication on nerve growth factor (NGF) signaling was also evaluated.

\section{Materials and methods}

Cell culture. The human salivary adenoid cystic carcinoma SACC-83 (Shanghai Jingkang Biotechnology) and human periodontal ligament fibroblast (HPLF) (Shanghai Zishi Biotechnology) cell lines were tested negative for mycoplasma and were authenticated by short tandem repeat markers. Cells were cultured in high-glucose Dulbecco's modified Eagle's medium (DMEM; Invitrogen; Thermo Fisher Scientific, Inc.) containing $10 \%$ fetal bovine serum (FBS; Gibco; Thermo Fisher Scientific, Inc.) and 1\% penicillin/streptomycin (Thermo Fisher Scientific, Inc.), and maintained at $37^{\circ} \mathrm{C}$ in a humidified incubator containing $5 \% \mathrm{CO}_{2}$.

Exosome isolation and fluorescence labeling. SACC-83 cells $\left(5 \times 10^{6}\right)$ were seeded and cultured in T-75 $\mathrm{cm}^{2}$ flask with DMEM complete medium until they reach $80 \%$ confluence and further grown for $24 \mathrm{~h}$ in serum-free medium. Cell conditioned medium was then collected to isolate exosomes using the Total Exosome Isolation Reagent kit (Invitrogen; Thermo Fisher Scientific, Inc.; cat. no. 4478359). Briefly, conditioned medium was centrifuged at $2,000 \mathrm{x} \mathrm{g}$ for $30 \mathrm{~min}$ at $4^{\circ} \mathrm{C}$ to remove cells and debris, and supernatant was collected, mixed with $5 \mathrm{ml}$ Total Exosome Isolation Reagent and incubated at $4^{\circ} \mathrm{C}$ overnight. The sample was centrifuged at $10,000 \mathrm{x}$ g for $1 \mathrm{~h}$ at $4^{\circ} \mathrm{C}$ and the subsequent exosome pellet was resuspended in $100 \mu \mathrm{l}$ PBS. The exosomes from five flasks of cells were pooled. The size and particle concentration of exosomes were then determined with the ZetaView nanoparticle tracking analysis (NTA; Particle Metrix Inc.). Exosomes were quantified with a BCA Protein Assay kit (Thermo Scientific) using an Absorbance Reader (ELX-800, BioTek Instruments, Inc.), labeled with PKH67 Green Fluorescent Cell Linker (Sigma-Aldrich; Merck KGaA; cat. no. PKH67GL) according to the manufacturer's protocol.

Observation of exosome internalization. HPLF cells $\left(5 \times 10^{5}\right)$ were incubated with various amounts of PKH67-labeled exosomes $(0,25,50$ or $75 \mu \mathrm{g})$ and for different incubation times $(0,3,6$ or $9 \mathrm{~h})$. Cell nucleus was stained with DAPI and cells were then fixed in $4 \%$ paraformaldehyde for $10 \mathrm{~min}$ at room temperature (RT). Cells were visualized with Zeiss LSM 510 confocal microscope (magnification, x630, Oberkochen, Germany).

Exosome visualization by transmission electron microscopy (TEM). Briefly, freshly isolated exosomes of which the density was $\sim 1.15 \mathrm{~g} / \mathrm{cm}^{3}$ were suspended in $2 \%$ paraformaldehyde over night at $4^{\circ} \mathrm{C}$, mounted on formvar carbon coated copper grids, fixed with $2 \%$ glutaraldehyde for $5 \mathrm{~min}$ and washed in sterile distilled water (11). Samples were dried and examined with a JEOL JEM-1010 transmission electron microscope (JEOL, Ltd.).

Western blotting. Exosomes or HPLF cell pellets were lysed in RIPA buffer containing $50 \mathrm{mM}$ Tris ( $\mathrm{pH} 7.4), 150 \mathrm{mM} \mathrm{NaCl}$, $1 \%$ Triton $\mathrm{X}-100,1 \%$ sodium deoxycholate and $0.1 \%$ SDS with protease inhibitor cocktail (Roche Applied Science), and quantified with a BCA Protein Assay kit (Thermo Scientific). Then, proteins $(15 \mu \mathrm{g})$ isolated from exosomes or cell lysate were separated by SDS-PAGE (10\% gel) and transferred onto polyvinylidene fluoride membranes (EMD Millipore). Membranes were blocked with 5\% skimmed milk in $1 \mathrm{X}$ Tris-buffered saline (TBS) containing $0.1 \%$ Tween-20 at RT for $2 \mathrm{~h}$, and incubated with primary antibodies against $\operatorname{CD} 63$ (1:1,000; Abcam; cat. no. ab59479), TSG101 (1:1,000; Abcam; cat. no. ab83), CD81 (1:1,000; Thermo Fisher Scientific Inc.; cat. no. 10630D), glucose-regulated protein 94 (GRP94; Cell Signaling Technology Inc.; cat. no. 20292), NGF (Abcam; cat. no. ab6199, NGF neutralizing antibody), or $\beta$-actin (Cell Signaling Technologies Inc.; cat. no. 4970) overnight at $4^{\circ} \mathrm{C}$. Membranes were washed and then incubated with horseradish peroxidase-conjugated secondary antibodies(1:50,000; goat anti-mouse; cat.no.115-035-146, or goat anti-rabbit; cat. no. 111-035-144; Jackson ImmunoResearch Inc.) for $1 \mathrm{~h}$ at RT. Reactive bands were detected with Immobilon ECL Ultra Western HRP Substrate (EMD Millipore). The relative intensities of the bands were quantified by densitometry and normalized to $\beta$-actin signal levels. Densitometric quantification was performed by Image-Pro Plus 6.0 software (Media Cybernetics, Inc. Rockville, MD, USA).

Cell invasion assay. SACC-83 cells $\left(1 \times 10^{5}\right)$ were suspended in the supernatant collected from PBS or exosomes-treated HPLF cells and placed into the upper chamber of Transwell cell culture insert (diameter, $6.5 \mathrm{~mm}$; pore size, $8.0 \mu \mathrm{m}$; Corning Inc.) of Matrigel (BD Biosciences) pre-coated 24-well plates. DMEM containing $10 \%$ FBS was added into the lower chamber. Following $24 \mathrm{~h}$ culture at $37^{\circ} \mathrm{C}$, SACC- 83 cells that have migrated onto the lower surface of the inserts were fixed in $4 \%$ paraformaldehyde for $30 \mathrm{~min}$ at RT, and stained with $0.1 \%$ crystal violet dissolved in PBS for 20 min at RT. Non-migrated cells at the bottom of the insert were removed and cells adherent to the lower surface of the insert were counted in six random fields using a light microscope at X100 magnification (Olympus IX5, Olympus America Inc.). The experiment was repeated at least three times.

Cell culture wound closure assay. SACC-83 cells were grown to $100 \%$ confluence in 12 -well plates and a scratch was generated with a sterile pipette tip. Cells were then incubated with the supernatant collected from PBS or exosomes-treated HPLF cells. Images were taken immediately $(0 \mathrm{~h})$ at x100 magnification following $24 \mathrm{~h}$ culture at $37^{\circ} \mathrm{C}$ using a light microscope (Olympus IX5). Cell migration ability was determined according to the percentage of area reduction or wound closure by Image-Pro Plus 6.0 software.

RNA isolation reverse transcription-quantitative polymerase chain reaction $(R T-q P C R)$. Total RNA was isolated from 
Table I. Sequences of primers used for for reverse transcription-quantitative polymerase chain reaction.

Genes Primer sequences

IL-6

$\begin{array}{ll}\text { Forward } & \text { 5'-TACATCCTCGACGCATCTC-3' } \\ \text { Reverse } & \text { 5'-AGCTCTGGCTGTTCCTCAC-3' }\end{array}$

IL-1 $\beta$

$\begin{array}{ll}\text { Forward } & \text { 5'-AAACAGATGAAGTGCTCCTTCCAGG-3' } \\ \text { Reverse } & \text { 5'-GTCGGAGATTCGTAGCTGGA-3' }\end{array}$

IL-8

Forward 5'-GAGAGTGATTGAGAGTGGACCAC-3'

Reverse 5'-CACAACCCTCTGCACCCAGTTT-3'

TNF- $\alpha$

$$
\text { Forward 5'-CCAGGCATCAGATCATCTTC-3' }
$$$$
\text { Reverse 5'-GGATGTTCGTCCTCCTCACAG-3' }
$$

MCP-1

$\begin{array}{ll}\text { Forward } & \text { 5'-CAGCCAGATGCAATCAATGCC-3' } \\ \text { Reverse } & \text { 5'-TGGAATCCTGAACCCACTTCT-3' }\end{array}$

NGF

Forward 5'-ACCCGCAACATTACTGTGGACC-3'

Reverse 5'-GACCTCGAAGTCCAGATCCTGA-3'

NTRK1

$\begin{array}{ll}\text { Forward } & \text { 5'-CACTAACAGCACATCTGGAGACC-3' } \\ \text { Reverse } & \text { 5'-TGAGCACAAGGAGCAGCGTAGA-3' } \\ \text { NTRK2 } & \\ \text { Forward } & \text { 5'-ACAGTCAGCTCAAGCCAGACAC-3' } \\ \text { Reverse } & \text { 5'-GTCCTGCTCAGGACAGAGGTTA-3' } \\ \text { NTF4 } & \\ \text { Forward } & \text { 5'-GCAAGGCTGATAACGCTGAGGA-3' } \\ \text { Reverse } & \text { 5'-CCTGGGCATCAGCGGTCAATG-3' }\end{array}$

CXCR4

Forward 5'-CTCCTCTTTGTCATCACGCTTCC-3'

Reverse 5'-GGATGAGGACACTGCTGTAGAG-3'

CXCL12

Forward 5'-CTCAACACTCCAAACTGTGCCC-3'

Reverse 5'-CTCCAGGTACTCCTGAATCCAC-3'

BDNF

$\begin{array}{lc}\text { Forward } & \text { 5'-CATCCGAGGACAAGGTGGCTTG-3' } \\ \text { Reverse } & \text { 5'-GCCGAACTTTCTGGTCCTCATC-3' } \\ \text { iNOS } & \\ \text { Forward } & \text { 5'-GCTCTACACCTCCAATGTGACC-3' } \\ \text { Reverse } & \text { 5'-CTGCCGAGATTTGAGCCTCATG-3' } \\ \text { MMP2 } & \\ \text { Forward } & \text { 5'-ATAACCTGGATGCCGTCGT-3' } \\ \text { Reverse } & \text { 5'-AGGCACCCTTGAAGAAGTAGC-3' } \\ \text { MMP9 } & \\ \text { Forward } & \text { 5'-GAACCAATCTCACCGACAGG-3' } \\ \text { Reverse } & \text { 5'-GCCACCCGAGTGTAACCATA-3' }\end{array}$

HPLF cells using TRIzol ${ }^{\circledR}$ modified Ultrapure RNA kit (CW Biotech) according to the manufacturer's instructions.
Table I. Continued.

\begin{tabular}{lc}
\hline Genes & Primer sequences \\
\hline GAPDH & \\
Forward & 5'-TTGCCATCAATGACCCCTTCA-3' \\
Reverse & 5'-CGCCCCACTTGATTTTGGA-3' \\
\hline
\end{tabular}

BDNF, Brain-derived neurotrophic factor; CXCL12, C-X-C motif chemokine ligand 12; CXCR4, C-X-C chemokine receptor type 4; GAPDH, glyceraldehyde 3-phosphate dehydrogenase; IL-1 $\beta$, interleukin $1 \beta$; IL-6, interleukin 6; IL-8, interleukin 8; iNOS, inducible nitroc oxide synthase; MCP-1, monocyte chemoattractant protein 1; MMP2, matrix metallopproteinase 2; MMP9, matrix metalloproteinase 9; NGF, nerve growth factor; NTF4, neurotrophin 4; NTRK1, neurotrophic receptor tyrosine kinase 1; NTRK2, neurotrophic receptor tyrosine kinase 2; TNF- $\alpha$, tumor necrosis factor $\alpha$.

The first strand of complementary DNA was synthesized using Oligo(dT) primer by SuperScript II reverse transcriptase (Invitrogen; Thermo Fisher Scientific, Inc.) according to the manufacturer's protocol. Subsequently, qPCRs were performed to analyze mRNA levels with SYBR Green PCR Master mix (Roche Applied Science) using StepOnePlus ${ }^{\mathrm{TM}}$ Real-Time PCR system (Thermo Fisher Scientific, Inc.). The PCR cycling conditions were $95^{\circ} \mathrm{C}$ for $30 \mathrm{sec}$ followed by 40 cycles of $95^{\circ} \mathrm{C}$ for $5 \mathrm{sec}$ and $60^{\circ} \mathrm{C}$ for $30 \mathrm{sec}$. The relative expression levels were normalized to the housekeeping gene GAPDH and calculated using the $2^{-\Delta \Delta C q}$ method (12). The sequences of the primers used in the present study are listed in Table I, and were synthesized by Thermo Fisher Scientific, Inc.

RNA sequencing and data analysis. HPLF cells were treated with PBS or SACC-83-derived exosomes. Total RNA was isolated using TRIzol ${ }^{\circledR}$ modified Ultrapure RNA kit (CW Biotech) and used for RNA sequencing analysis. The complementary DNA library was constructed and sequenced by BGI Genomics Services using BGISEQ-500 platform. Bioinformatics workflow including data filtering, mapping transcript prediction, differential gene expression analysis, gene ontology (GO; http://geneontology.org) and Kyoto Encyclopedia of Genes and Genomes (KEGG; https://www. genome.jp/kegg) pathway analysis were performed by procedures established by BGI Genomics Services. The in-depth bioinformatics analysis of NTRK1 expression in the Oncomine database was carried out at https://www.oncomine.org/.

Cytokine secretion measurement. Human $\beta$-NGF secreted by HPLF cells in culture supernatants was quantified using ELISA (cat. no. DY256-05; R\&D Systems, Minneapolis, MN, USA), according to the manufacturer's instructions.

Immunohistochemistry (IHC) analyses. Human SACC tissues were obtained by the Changhai Hospital. The SACC patients were treated in the Hospital between January 2016 and May 2018, and diagnosed with a surgical biopsy by histopathology. The mean age was 56 years (range, 50-65 years). All patients provided written informed consent, and the use of human samples was approved by the Shanghai Changhai 

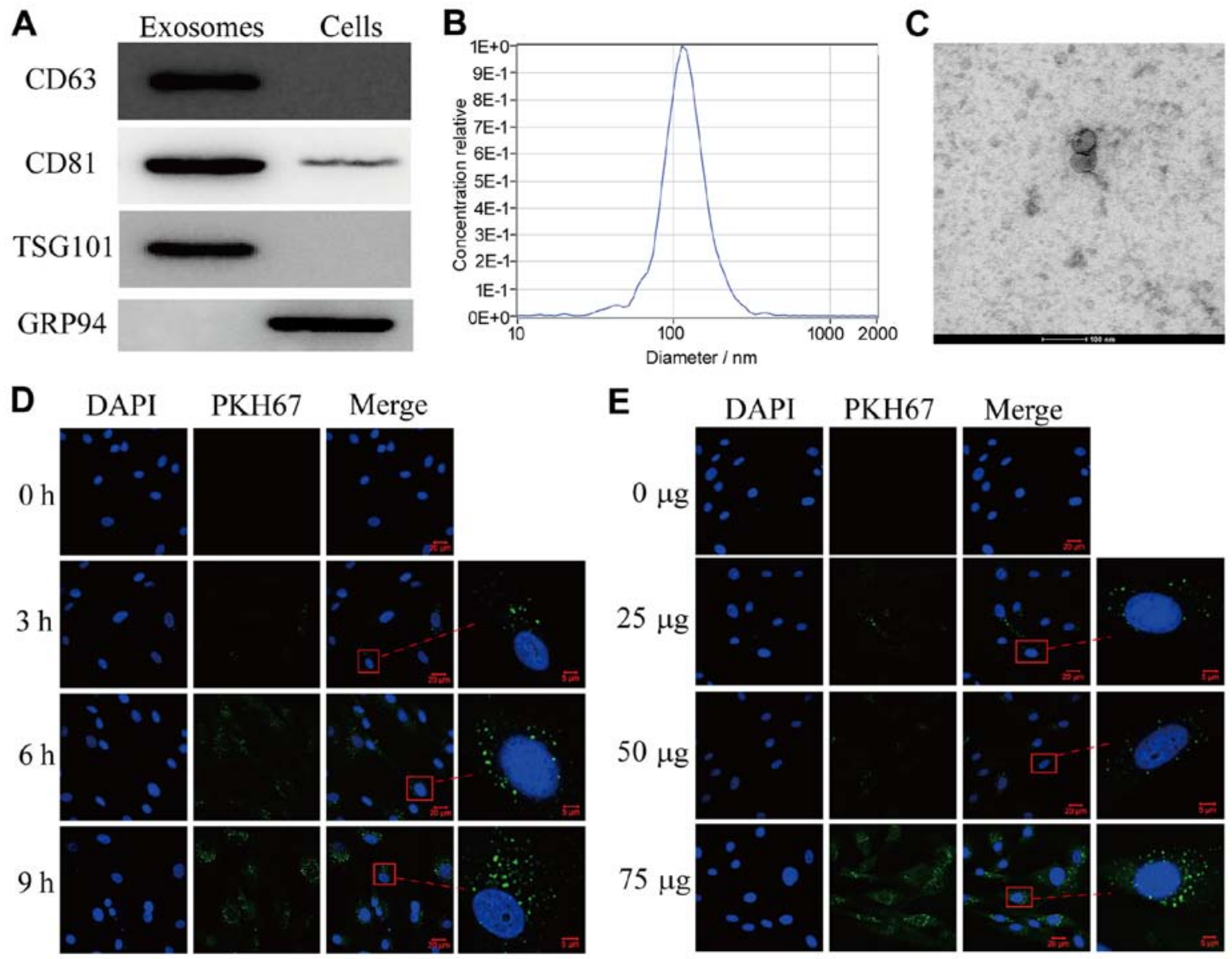

Figure 1. Exosomes purified from SACC-83 cells are delivered into HPLF cells. (A) Western blotting analysis showing the presence of CD63, CD81, TSG101 and the absence of GRP94 in SACC-83 cell-derived exosomes. (B) Nanoparticle tracking analysis and (C) transmission electron microscopy images showing exosomes isolated from SACC-83 cells. Magnification, x630; scale bar, $20 \mu \mathrm{m}$. (D) Confocal microscopy images showing the internalization of PKH67-labeled exosomes in HPLF cells at different incubation times. Scale bar, $5 \mu \mathrm{m}$. (E) Confocal microscopy images showing the delivery of $0,25,50$ or $75 \mu \mathrm{g}$ of exosomes into HPLF cells after $6 \mathrm{~h}$. Scale bar, $5 \mu \mathrm{m}$. Magnified views are shown on the right. GRP94, glucose-regulated protein 94; HPLF, human periodontal ligament fibroblast; SACC, salivary adenoid cystic carcinoma.

Hospital Ethics Committee. Tissues were fixed in $10 \%$ formalin for $24 \mathrm{~h}$ at RT and embedded in paraffin. Tissue sections (5 $\mu \mathrm{m}$-thick) were cut for IHC staining. The antigen retrieval was performed using a pressure cooker at $121^{\circ} \mathrm{C}$. Endogenous peroxidase activity was blocked by $3 \%$ hydrogen peroxide at RT for 20 min. Normal goat serum (10\%; CW Biotech) was used to block nonspecific binding at RT for $1 \mathrm{~h}$. Sections were incubated with NTRK1 rabbit monoclonal primary antibody (Abcam; cat. no. ab76291; 1:100; clone, EP1058Y) at $4^{\circ} \mathrm{C}$ overnight. The subsequent procedures were performed using a SP Rabbit HRP kit (DAB) (cat. no. CW2035, CW Biotech) in accordance with the manufacturer's protocol. The tissue sections were counterstained by immersing sides in hematoxylin for 2 min at RT. Antibody staining was then observed under a light microscope at x100, x200 and x400 magnification (Olympus IX5).

Statistical analysis. Two-tailed Student's t-test was used to compare two groups. Comparison among multiple groups was performed using one-way analysis of variance followed by Tukey's post hoc test of significance between individual groups. Data are presented as the means \pm standard error of the mean. Statistical analyses were performed with GraphPad
Prism (v7.0; GraphPad Software, Inc.). $\mathrm{P}<0.05$ was considered to indicate a statistically significant difference. Each experiment was repeated at least three times.

\section{Results}

SACC-derived exosomes are efficiently internalized into fibroblasts. Exosomes were isolated from conditioned medium of SACC-83 cells. As presented in Fig. 1A, exosomes were characterized by the three specific exosome markers CD63, CD81 and TSG101 (13); however, GRP94 was absent. Furthermore, the size and concentration $\left(\sim 8.2 \times 10^{10}\right.$ particles $\left./ \mathrm{ml}\right)$ of exosomes were determined by NTA (Fig. 1B) and TEM (Fig. 1C). The results demonstrated that exosome diameter ranged between 100 and $150 \mathrm{~nm}$. To examine the transfer ability of exosomes to HPLF cells, SACC-83-derived exosomes were labeled with PKH67 and incubated with HPLF cells for 3, 6 or $9 \mathrm{~h}$ (Fig. 1D). The results from confocal microscopy analysis demonstrated that green fluorescent exosomes were internalized into HPLF cells in a time- and concentration-dependent manner (Fig. 1D and E, respectively). These results indicated that SACC-83-derived exosomes may be transferred into HPLF cells. 
A
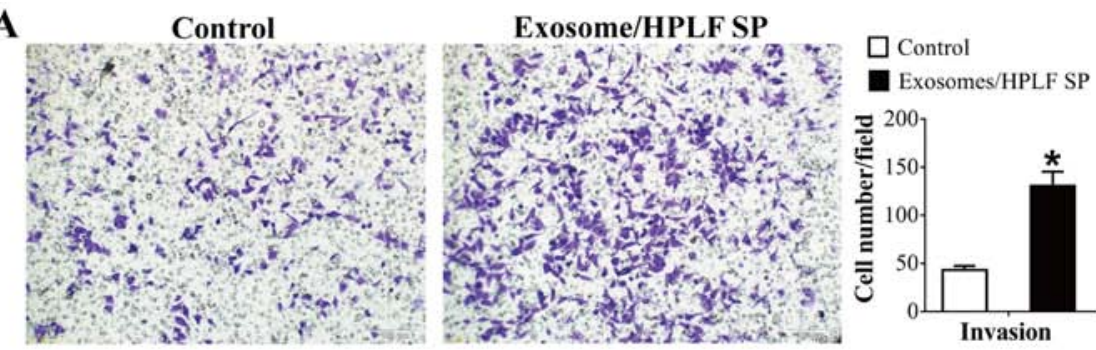

B
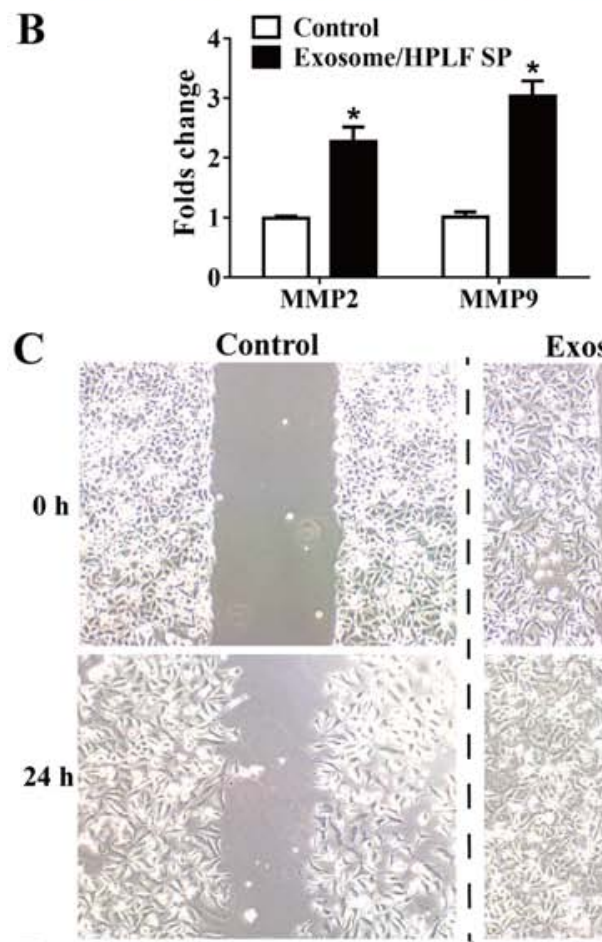

D

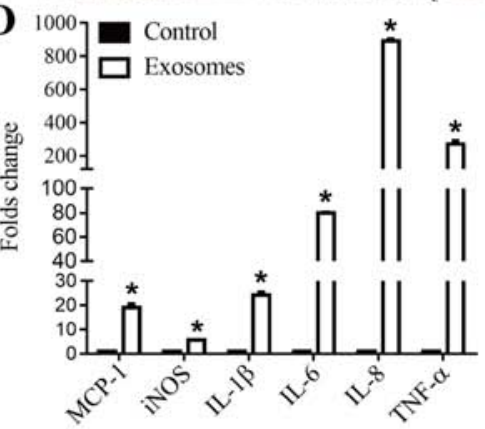

Exosome/HPLF SP
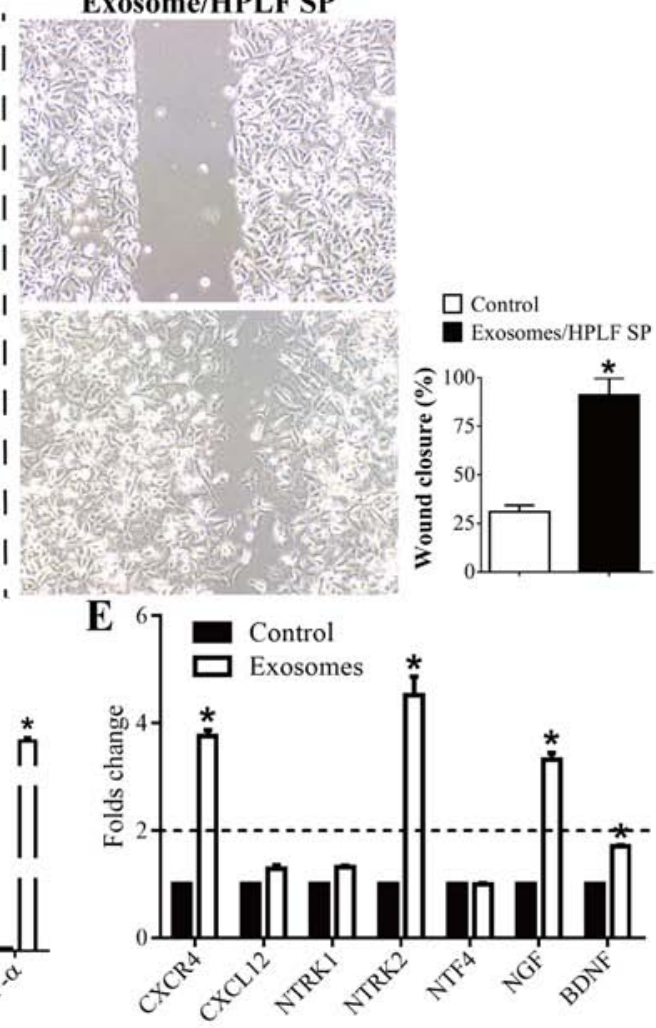

Figure 2. Exosomes stimulated HPLF cells to facilitate SACC-83 cell invasion and enhance the expression of proinflammatory and perineural mediators in HPLF cells. (A) Transwell assay was used to assess the invasive ability of SACC-83 cells treated with control or the culture SP from exosome-educated HPLF cells (Exosome/HPLF SP). (B) RT-qPCR for analysis of MMP2 and MMP9 gene expression levels in SACC-83 cells. (C) SACC-83 cell migration was determined using wound-healing assay. (D) RT-qPCR for analysis of MCP-1, iNOS, IL-1 $\beta$, IL-6, IL-8 and TNF- $\alpha$ gene expression levels in HPLF cells. (E) RT-qPCR for analysis of CXCR4, CXCL12, NTRK1, NTRK2, NTF4, NGF and BDNF gene expression levels in HPLF cells. Data represent the mean \pm standard error of the mean of at least three independent experiments. "P $<0.05$ vs. control group. BDNF, Brain-derived neurotrophic factor; CXCL12, C-X-C motif chemokine ligand 12; CXCR4, C-X-C chemokine receptor type 4; GAPDH, glyceraldehyde 3-phosphate dehydrogenase; HPLF, human periodontal ligament fibroblast; IL-1 $\beta$, interleukin 1 $\beta$; IL-6, interleukin 6; iNOS, inducible nitroc oxide synthase; MCP-1, monocyte chemoattractant protein 1; MMP2, matrix metalloproteinase 2; MMP9, matrix metalloproteinase 9; NGF, nerve growth factor; NTF4, neurotrophin 4; NTRK1, neurotrophic receptor tyrosine kinase 1; NTRK2, neurotrophic receptor tyrosine kinase 2; TNF- $\alpha$, tumor necrosis factor $\alpha$; RT-qPCR, reverse transcription-quantitative polymerase chain reaction; SACC, salivary adenoid cystic carcinoma; SP, supernatant.

Tumor-derived exosomes stimulate HPLF cells to facilitate SACC-83 cell migration and enhance the expression of proinflammatoryand perineural mediators in HPLF cells. Exosomes secreted from cells can transfer biological information to other recipient cells and may therefore regulate the crosstalk between cancer cells and tumor microenvironment (14). The present study investigated whether the administration of
SACC-83-derived exosomes to HPLF cells would result in HPLF cell change of phenotype, and subsequently influence the metastasis of SACC- 83 cells. The results demonstrated that SACC-83 exhibited a higher invasive ability following stimulation with the supernatant from exosome-educated HPLF cells compared with control (Fig. 2A). Furthermore, the expression levels of matrix metalloproteinases 2 and 9 (MMP2 
A

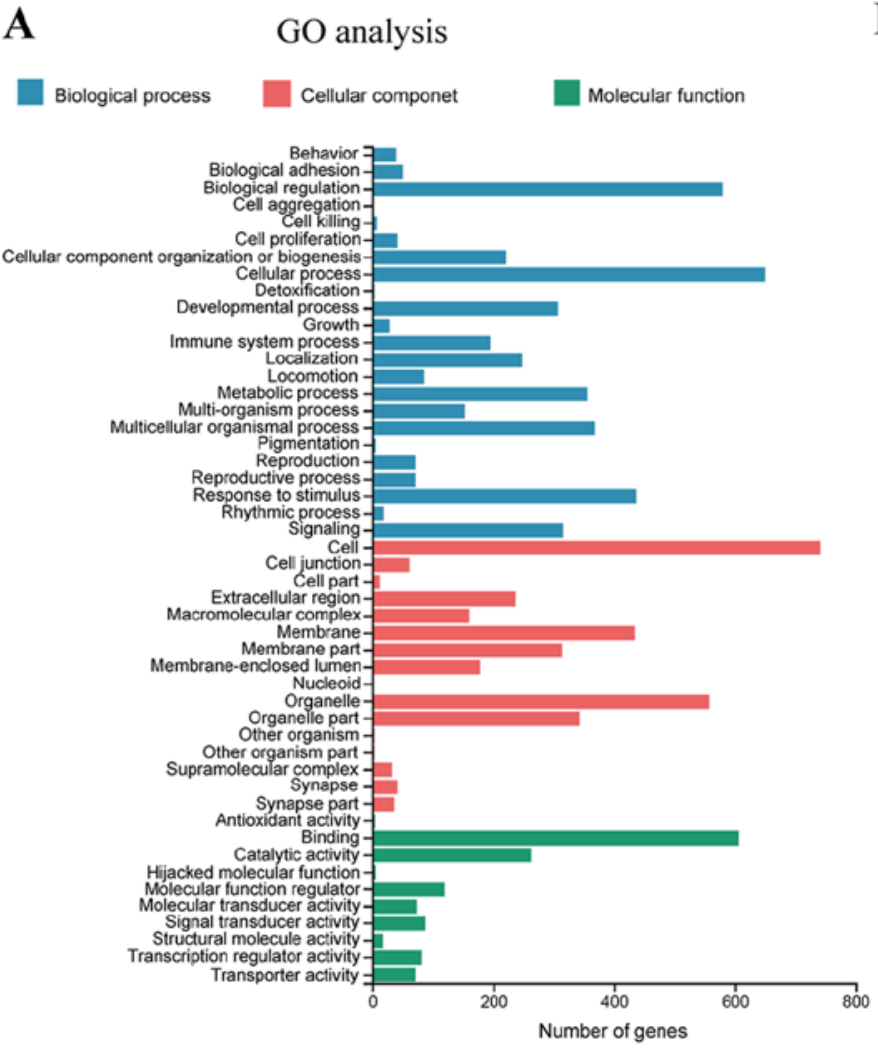

GO enrichment bubble chart

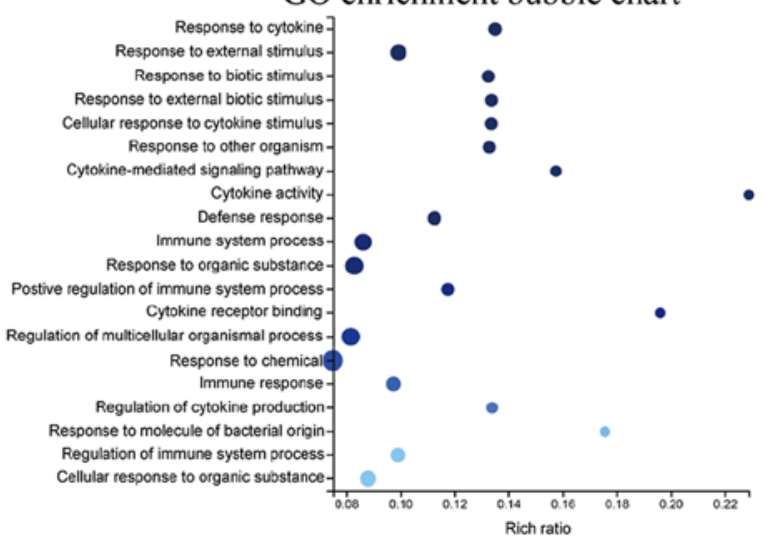

C norm

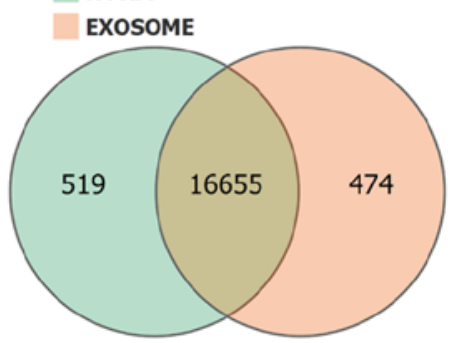

B

KEGG pathway analysis

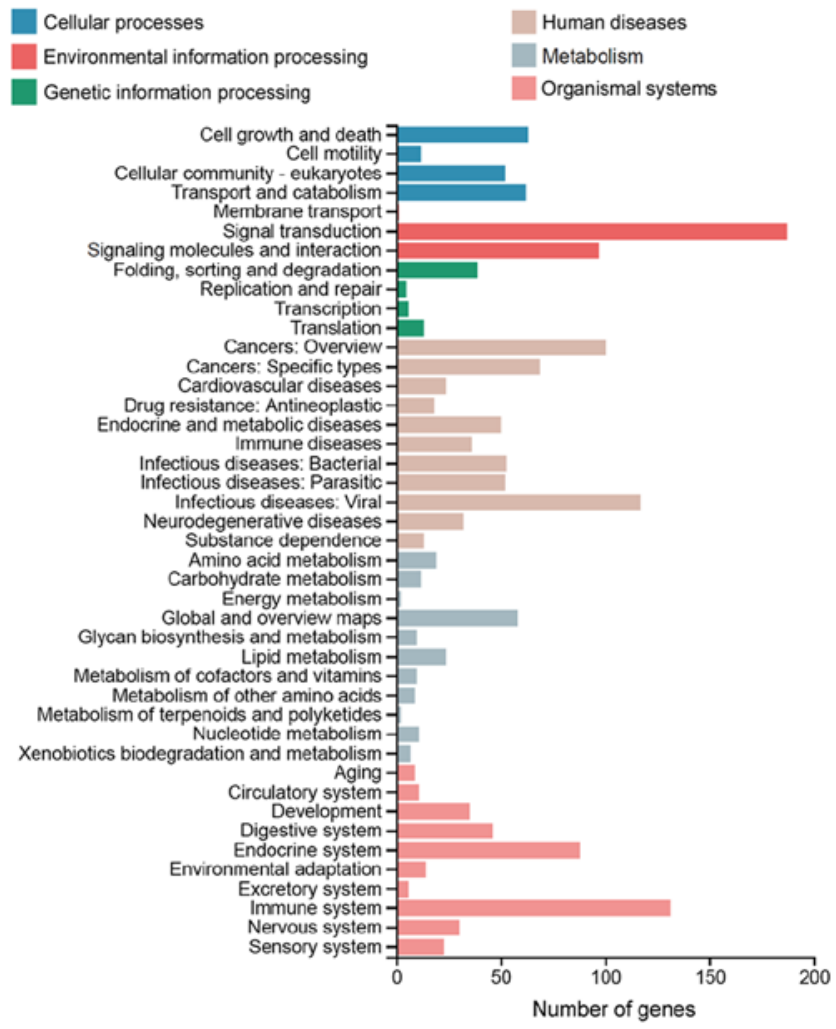

KEGG enrichment bubble chart

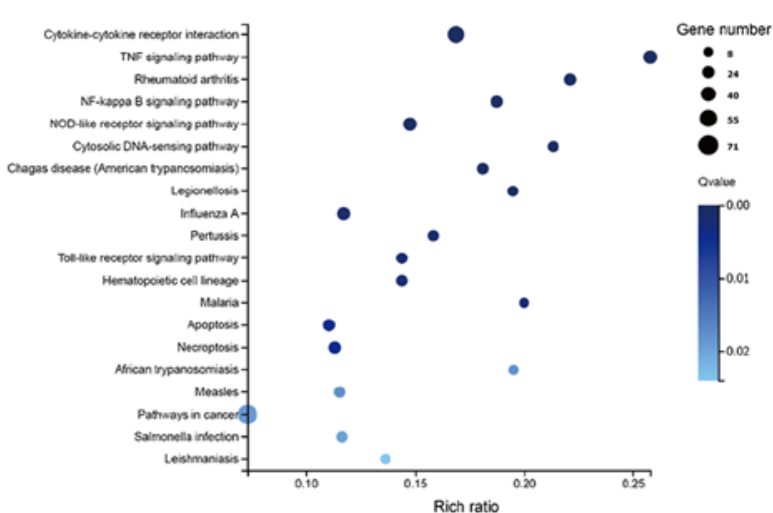

$\mathbf{E}$
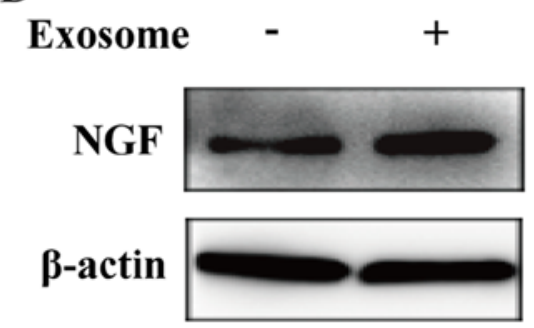
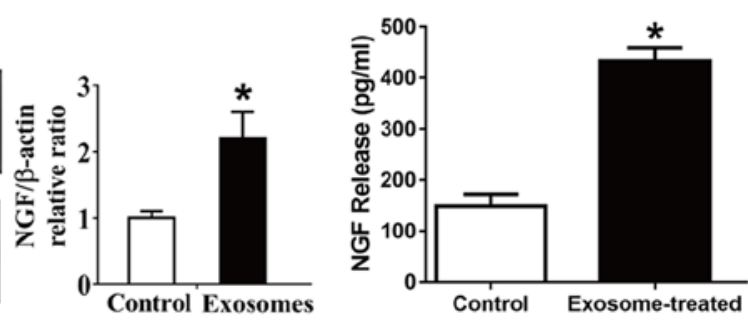

Figure 3. Exosomes mediate the protumorigenic effect of HPLF cells and enhance their NGF expression. (A) GO-term analysis of altered genes expression in exosome-educated HPLF cells. (B) KEGG pathway analysis of altered genes in exosome-treated HPLF cells. (C) Venn diagram showing the overlap of altered genes in control and exosome-treated HPLF cells. (D) NGF protein level was determined in control or exosomes-treated HPLF cells by western blotting. (E) NGF release was measured in the culture media from control or exosomes-treated HPLF cells by ELISA. Data represent the mean \pm standard error of the mean of at least three independent experiments. "P<0.05 vs. control group. HPLF, human periodontal ligament fibroblast; GO, gene ontology; KEGG, Kyoto Encyclopedia of Genes and Genomes; NGF, nerve growth factor.

and MMP9), which are responsible for promoting cancer invasion (15), were significantly increased compared with control (Fig. 2B). Consistent with the invasion assay results, SACC-83 cells migration was significantly increased following stimulation with the culture medium collected from exosome-educated HPLF cells (Fig. 2C). To determine the expression of common 

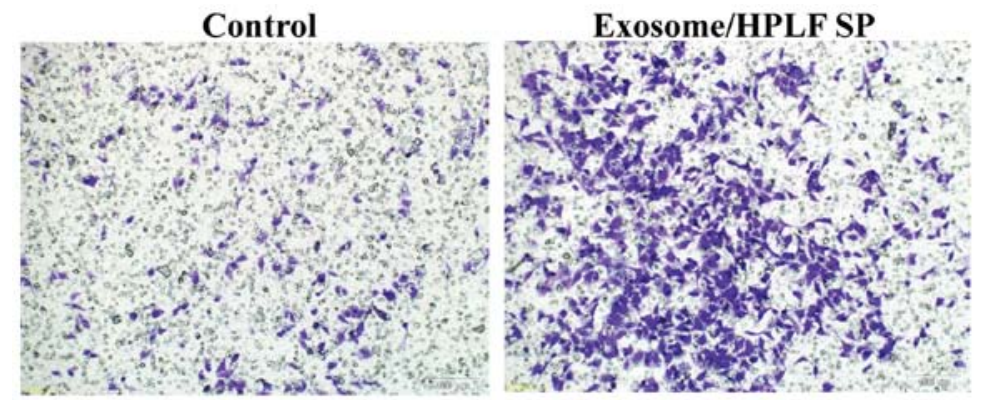

Exosome/HPLF SP+NGF Ab
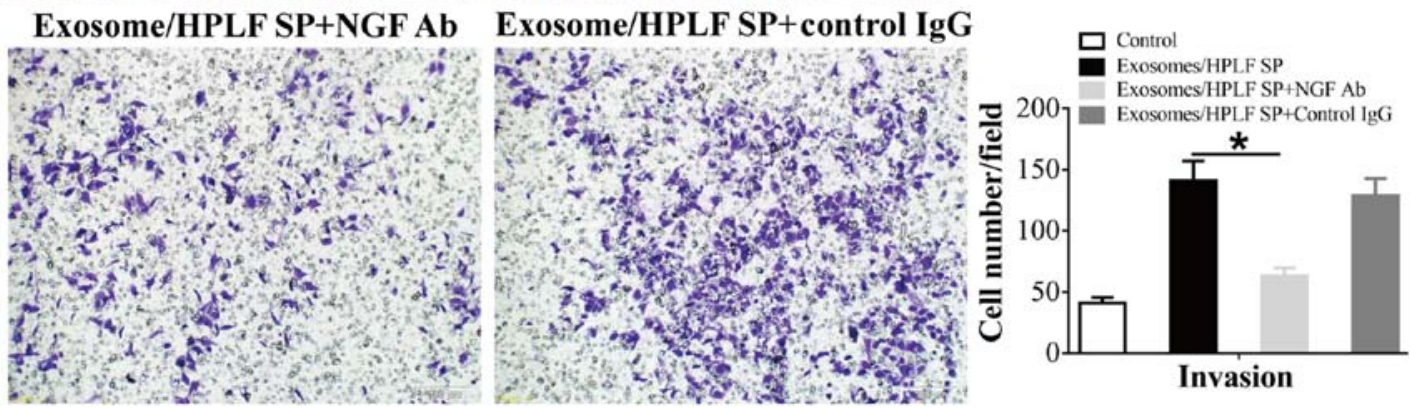

Figure 4. Neutralizing NGF reduces the invasive ability of SACC- 83 cells that are stimulated by the SP collected from exosome-educated HPLF cells (Exosome/HPLF SP). The functional NGF neutralizing Ab or control $\operatorname{IgG~}(10 \mu \mathrm{g} / \mathrm{ml})$ was used in the Transwell assay. Data represent the mean \pm standard error of the mean of at least three independent experiments. "P $<0.05$ vs. control group. Ab, antibody; IgG, immunoglobulin G; HPLF, human periodontal ligament fibroblast; SACC, salivary adenoid cystic carcinoma.

proinflammatory factors in exosome-educated HPLF cells, RT-qPCR was performed to detect the mRNA levels of monocyte chemoattractant protein 1 (MCP-1), inducible nitric oxide synthase (iNOS), interleukin (IL)-6 $1 \beta$ and 8 and tumor necrosis factor $\alpha$ (TNF- $\alpha$ ). As presented in Fig. 2D, the expression levels of MCP-1, iNOS, IL-6, IL-1 $\beta$, IL-8 and TNF- $\alpha$ were significantly increased in exosomes-treated HPLF cells compared with PBS-treated HPLF cells. Furthermore, the expression levels of factors involved in tumor metastasis and PNI, including C-X-C chemokine receptor type 4 (CXCR4), $\mathrm{C}-\mathrm{X}-\mathrm{C}$ motif chemokine ligand 12 (CXCL12), neurotrophic receptor tyrosine kinases (NTRK) 1 and 2, neurotrophin 4 (NTF4), NGF and brain-derived neurotrophic factor (BDNF) were examined. The results demonstrated that the expression levels of CXCR4, NTRK2, NGF and BDNF were significantly increased in exosome-educated HPLF cells compared to those in PBS-treated HPLF cells (Fig. 2E).

Exosomes mediate the pro-tumorigenic effects of HPLF cells and enhance NGF expression in HPLF cells. In order to identify how the culture supernatant from exosome-treated HPLF cells could enhance SACC-83 cell invasion ability, an unbiased RNA sequencing analysis was performed to determine the transcriptome profile of PBS or exosome-treated HPLF cells. The average RNA sequencing depth was 21.89 million reads, with an average genome mapping rate of $93.67 \%$, and the total number of genes identified was 17,648. Furthermore, 943 differentially expressed genes with $>2$-fold expression change were identified. GO term analysis of these altered genes exhibited enrichment in the 'response to chemical' and 'immune system process' (Fig. 3A). The results from KEGG pathway analysis reported enrichment in 'cytokine-cytokine receptor interaction' (Fig. 3B). There were 16,655 genes expression in both PBS or exosome-treated HPLF cells, whereas 519 genes were expressed only in PBS-treated HPLF cells, and 474 genes were expressed only in exosome-treated HPLF cells (Fig. 3C). The expression of NGF, which was proposed as a potential prognosis and pathogenic biomarker of SACC (16-18), was then examined. As presented in Fig. 3D and E, NGF protein expression was significantly increased in exosome-educated HPLF cells.

Neutralizing NGF reduces SACC-83 cell invasion stimulated by the supernatant from exosome-treated HPLF cells. In order to determine whether the enhanced SACC- 83 cell migratory ability was caused by NGF released from exosome-treated HPLF cells, the functional NGF neutralizing antibody was used in the invasion assay. As presented in Fig. 4, NGF blockage significantly reduced the enhanced invasive ability of SACC-83 cells following treatment with supernatant from exosome-educated HPLF cells.

Upregulation of NTRK1 expression in patients with SACC. To illustrate the roles of NGF signaling in SACC progression, in-depth bioinformatics analysis of the Oncomine database was carried out. The results demonstrated that NTRK1 overexpression ranked in the top $1 \%$ of increased gene expression in salivary gland adenoid cystic carcinoma vs. normal (Fig. 5A), and that NTRK1 mRNA level was increased by a 28.356 -fold in SACC tissue compared to normal tissue from the Oncomine database (19) (Fig. 5A). Furthermore, NTRK1 expression was examined by IHC in tumor tissues from patients with SACC. As presented in Fig. 5B, the cancerous tissues staining revealed strongly positive NTRK1 expression, whereas the non-cancerous tissues staining (red arrow) revealed negative NTRK1 expression. The protein expression of NTRK1 was markedly upregulated in the cancerous tissues from three patients with SACC. 
A

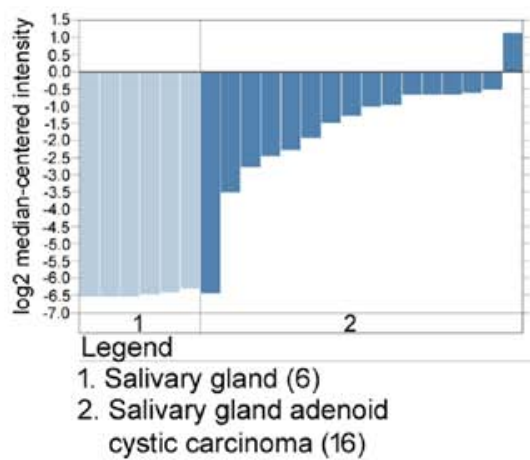

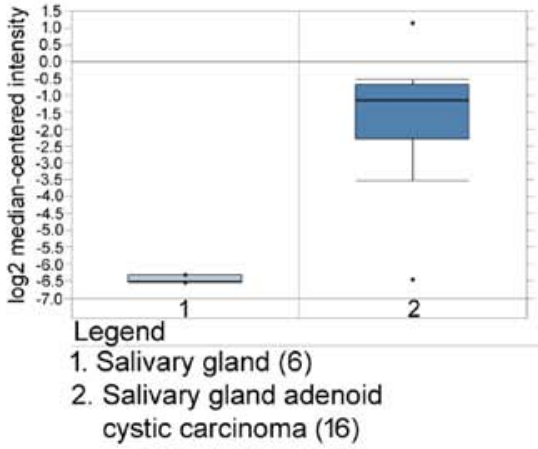

cystic carcinoma (16)

NTRK1 expression in FriersonHF salivary gland

Salivary gland adenoid cystic carcinoma vs. normal Over-expression gene rank: 41(in top 1\%)

Reporter: 36805 s_at $\mathbf{V}$

P-value:3.85E-9

T-test: 11.354
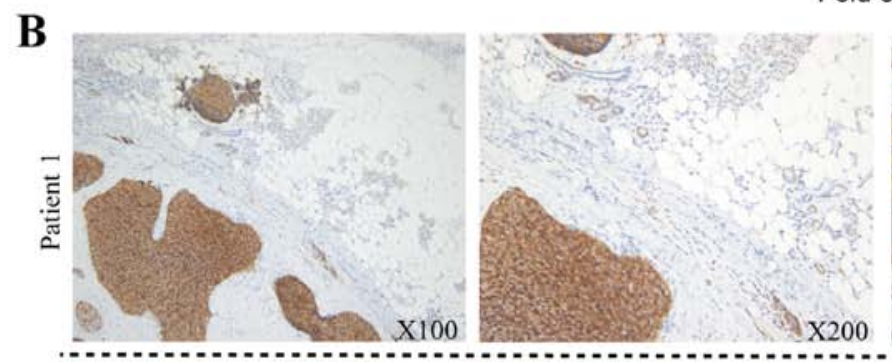

Fold change: 28.356
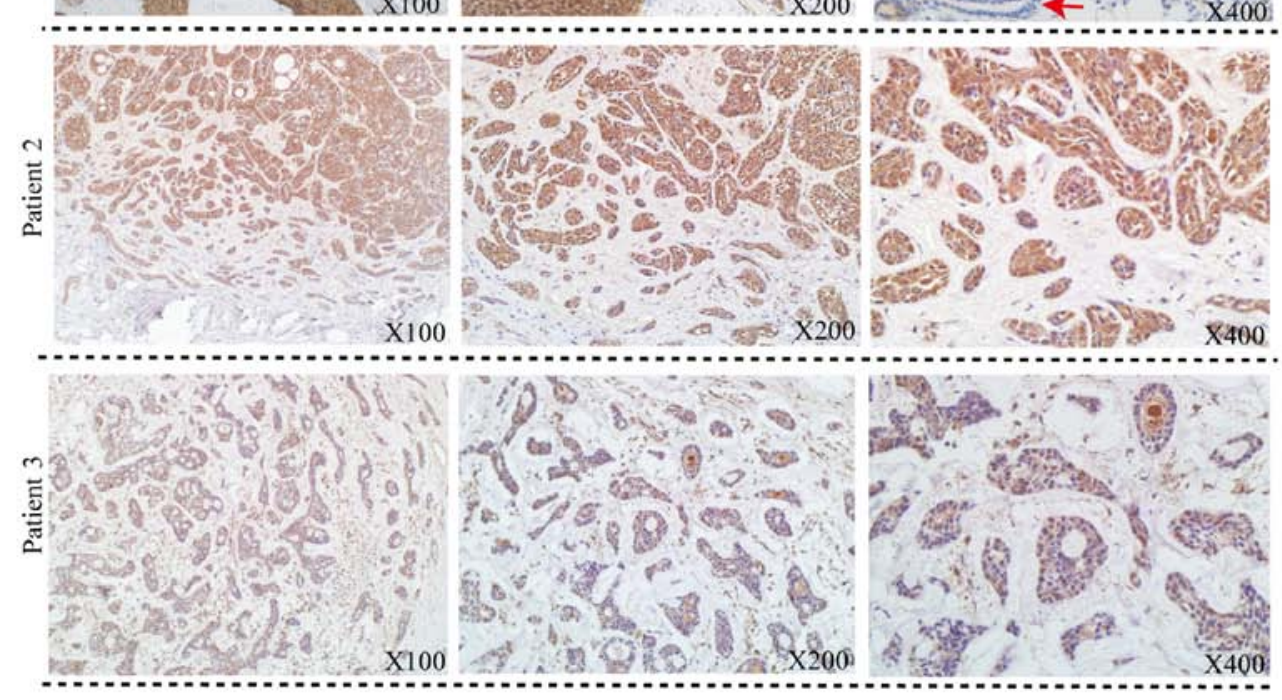

Figure 5. Overexpression of NGF receptor NTRK1 in patients with SACC. (A) Oncomine analysis of NTRK1 expression in SACC samples. "P<0.05 vs. normal samples. (B) NTRK1 expression was determined in tissue samples from three representative patients with SACC by immunohistochemistry staining analysis. The red arrows indicate non-cancerous tissues from the patient with SACC. NGF, nerve growth factor; NTRK1, neurotrophic receptor tyrosine kinase 1; SACC, salivary adenoid cystic carcinoma.

\section{Discussion}

SACC is a rare type of head and neck cancer characterized by unpredictable growth, extensive PNI and high risk of metastasis. PNI corresponds to the neoplastic invasion of nerves along with increased recurrence rates and reduced survival $(2,3)$.Although the clinical significance of PNI is widely recognized, its underlying mechanisms remain unclear. Factors involved in nerve microenvironment, including chemokines, neurotrophic factors and cellular adhesion molecules, have recently gained more attention (20). Cancer-associated fibroblasts are localized within the tumor microenvironment and produce certain mediators, including growth factors, chemokines and extracellular matrix that enhance cancer cell metastasis (21). CAFs can therefore provide a unique tumor microenvironment that stimulates cancer development and progression (22). Perineurium originates from the differentiation of fibroblasts (21) and any change in composition and malfunction of the perineurium can lead to tumor invasion (20). The role of fibroblast in tumor metastasis and PNI requires therefore more attention.

In the present study, the crosstalk between human SACC cells and fibroblasts was investigated. Cell-derived exosomes act as a bridge between cells to deliver molecules and link cellular functions. It has been demonstrated that pancreatic fibroblast-derived exosomes promote the proliferation and 
survival of cancer cells (23). A recent study reported that CAFs account for oral squamous cell carcinoma progression and metastasis through exosome-derived paracrine miR-34a-5p (24). This study therefore investigated the role of tumor-derived exosome-educated HPLF cells in SACC-83 cell metastasis. The results demonstrated that supernatant from exosome-educated HPLF cells markedly promoted SACC-83 invasion, which suggested that exosomes may be considered as key mediators of the tumorigenic effect of fibroblasts on SACC pathogenesis.

To comprehensively understand the underlying mechanisms by which tumor-derived exosomes influenced fibroblast functions and subsequently SACC progression, RNA sequencing analysis was performed in order to analyze the gene expression changes in exosome-treated HPLF cells. A total of 943 differentially expressed genes (upregulated or downregulated) with $>2$-fold expression change were identified, and these genes exhibited enriched cytokine-cytokine receptor interaction. Chronic inflammation, which has been recognized as one of the major tumor hallmarks, can influence almost all stages of tumorigenesis (25). The present study demonstrated that the expression levels of the proinflammatory cytokines MCP-1, IL-1 $\beta$, IL-6, IL- 8 and TNF- $\alpha$ were significantly increased in exosome-stimulated HPLF cells. These inflammatory factors, including IL-1 $\beta$, have been reported to serve a crucial role in the proliferation, migration and invasion of malignant tumor cells $(26,27)$. The results from the present study suggested that SACC-83 cell-derived exosomes may educate HPLF cells to create an inflammatory microenvironment that could facilitate tumor progression.

Chemokines and their receptors can directly affect cancer cell proliferation and movement or indirectly modulate tumor growth by stimulating the secretion of growth, chemotactic and angiogenic factors from stromal cells into the cancer microenvironment (28). For example, nerve-derived $\mathrm{C}-\mathrm{C}$ motif chemokine ligand 2 facilitates prostate cancer cell invasion and PNI via $\mathrm{C}-\mathrm{C}$ chemokine receptor type 2-mediated signaling (29). Furthermore, CXCL12 can facilitate squamous cell/carcinoma cell survival and migration via its receptor CXCR4 (30). The results from the present study demonstrated that expression levels of CXCR4, NTRK2, NGF and BDNF were significantly increased in exosome-stimulated HPLF cells. It has been demonstrated that NGF and its receptor NTRK1 are associated with metastasis and PNI in several types of cancer. For example, expression levels of NGF and its high and low affinity receptors NTRK1 and p75NTR, respectively, were markedly elevated in pancreatic cancer cells and surrounding nerves, which was positively correlated with the presence of PNI (31). Furthermore, in oral tongue tumor tissues, stronger NGF and NTRK1 staining was observed in tissues with PNI compared with tissues of the same stage but without PNI (32). The present study demonstrated that NGF protein level was significantly increased in exosome-educated HPLF cells. In particular, mRNA levels of NTRK1 in HPLF cells did not change following exosome treatment. Furthermore, NGF blockage significantly reduced the enhanced invasive ability of SACC- 83 cells following treatment with culture medium from exosome-educated
HPLF cells. Cancer cell-derived exosomes can be internalized into the recipient cells and regulate their biological function and signaling (33). Similarly, the results from this study indicated that exosomes may serve a crucial role in tumor microenvironment and enhance the crosstalk between SACC-83 cells and HPLF cells. Furthermore, results from the Oncomine database analysis demonstrated that NTRK1 overexpression ranked in the top $1 \%$ of increased gene expression in SACC tissues compared with normal tissues. Consistent with this finding, higher expression level of NTRK1 was observed in the cancerous tissues compared with in non-cancerous tissues from patients with SACC.

In conclusion, the present study demonstrated that SACC-83 cell-derived exosomes stimulated HPLF cells to produce NGF, which may enhance SACC-83 cell invasion. The results also indicated that tumor-derived exosomes may lead to HPLF cell activation and downstream inflammation. This mechanism may illustrate the crosstalk between SACC and fibroblasts and explain the occurrence of metastasis and PNI in SACC. Since NGF-NTRK1 signaling results in poor prognosis in patients with SACC (18), targeting tumor-derived exosomes and CAFs by blocking NGF-NTRK1 pathway may potentially serve as a novel therapy that could be used in synergy with conventional therapies in order to improve patient survival.

\section{Acknowledgements}

Not applicable.

\section{Funding}

This study was supported by the National Natural Science Foundation of China (grant nos. 81772577 and 81602497).

\section{Availability of data and materials}

All data generated or analyzed during this study are included in this published article.

\section{Authors' contributions}

ZX and JZ designed the study and wrote the manuscript. ZX and XZ performed the experiments. All authors read and approved the final manuscript.

\section{Ethics approval and consent to participate}

All procedures performed in studies involving human tissues were in accordance with the ethical standards of the Institutional Committee and with the Declaration of Helsinki. All patients provided written informed consent prior to the study start. Use of human samples in the present study was approved by Shanghai Changhai Hospital Ethics Committee.

\section{Patient consent for publication}

Not applicable. 


\section{Competing interests}

The authors declare that they have no competing interests.

\section{References}

1. Venteicher AS, Walcott BP, Sheth SA, Snuderl M, Patel AP, Curry WT and Nahed BV: Clinical features of brain metastasis from salivary gland tumors. J Clin Neurosci 20: 1533-1537, 2013.

2. Ellington CL, Goodman M, Kono SA, Grist W, Wadsworth T, Chen AY, Owonikoko T, Ramalingam S, Shin DM, Khuri FR, et al: Adenoid cystic carcinoma of the head and neck: Incidence and survival trends based on 1973-2007 Surveillance, Epidemiology and End Results data. Cancer 118: 4444-4451, 2012.

3. Ho AS, Kannan K, Roy DM, Morris LG, Ganly I, Katabi N, Ramaswami D, Walsh LA, Eng S, Huse JT, et al: The mutational landscape of adenoid cystic carcinoma. Nat Genet 45: 791-798, 2013.

4. Shiga K, Hara M, Nagasaki T, Sato T, Takahashi H and Takeyama H: Cancer-associated fibroblasts: Their characteristics and their roles in tumor growth. Cancers 7: 2443-2458, 2015.

5. Madar S, Goldstein I and Rotter V: 'Cancer associated fibroblasts'-more than meets the eye. Trends Mol Med 19: 447-453 2013.

6. Liotta LA and Kohn EC: The microenvironment of the tumour-host interface. Nature 411: 375-379, 2001.

7. Xing F, Saidou J and Watabe K: Cancer associated fibroblasts (CAFs) in tumor microenvironment. Front Biosci (Landmark Ed) 15: 166-179, 2010.

8. Che Y, Geng B, Xu Y, Miao X, Chen L, Mu X, Pan J, Zhang C, Zhao T, Wang C, et al: Helicobacter pylori-induced exosomal MET educates tumour-associated macrophages to promote gastric cancer progression. J Cell Mol Med 22: 5708-5719, 2018.

9. Zhang H, Deng T, Liu R, Bai M, Zhou L, Wang X, Li S, Wang X, Yang H, Li J, et al: Exosome-delivered EGFR regulates liver microenvironment to promote gastric cancer liver metastasis. Nat Commu 8: 15016, 2017.

10. Ringuette Goulet C, Bernard G, Tremblay S, Chabaud S, Bolduc $S$ and Pouliot F: Exosomes induce fibroblast differentiation into cancer-associated fibroblasts through TGF $\beta$ signaling. Mol Cancer Res 16: 1196-1204, 2018.

11. Théry C, Amigorena S, Raposo G and Clayton A: Isolation and characterization of exosomes from cell culture supernatants and biological fluids. Curr Protoc Cell Biol Chapter 3: Unit 3.22, 2006.

12. Livak KJ and Schmittgen TD: Analysis of relative gene expression data using real-time quantitative PCR and the 2(-Delta Delta C(T)) method. Methods 25: 402-408, 2001.

13. Andreu $\mathrm{Z}$ and Yáñez-Mó M: Tetraspanins in extracellular vesicle formation and function. Front Immunol 5: 442, 2014.

14. Ruivo CF, Adem B, Silva M and Melo SA: The biology of cancer exosomes: Insights and new perspectives. Cancer Res 77: 6480-6488, 2017.

15. Deryugina EI and Quigley JP: Matrix metalloproteinases and tumor metastasis. Cancer Metastasis Rev 25: 9-34, 2006.

16. Hao L, Xiao-Lin N, Qi C, Yi-Ping Y, Jia-Quan L and Yan-Ning L: Nerve growth factor and vascular endothelial growth factor: Retrospective analysis of 63 patients with salivary adenoid cystic carcinoma. Int J Oral Sci 2: 35-44, 2010.

17. Wang L, Sun M, Jiang Y, Yang L, Lei D, Lu C, Zhao Y, Zhang P, Yang $\mathrm{Y}$ and Li J: Nerve growth factor and tyrosine kinase A in human salivary adenoid cystic carcinoma: Expression patterns and effects on in vitro invasive behavior. J Oral Maxillofac Surg 64: 636-641, 2006.
18. Kobayashi K, Ando M, Saito Y, Kondo K, Omura G, Shinozaki-Ushiku A, Fukayama M, Asakage T and Yamasoba T: Nerve growth factor signals as possible pathogenic biomarkers for perineural invasion in adenoid cystic carcinoma. Otolaryngol Head Neck Surg 153: 218-224, 2015.

19. Frierson HF Jr, El-Naggar AK, Welsh JB, Sapinoso LM, Su AI, Cheng J, Saku T, Moskaluk CA and Hampton GM: Large scale molecular analysis identifies genes with altered expression in salivary adenoid cystic carcinoma. Am J Pathol 161: 1315-1323, 2002.

20. Bakst RL and Wong RJ: Mechanisms of perineural invasion. J Neurol Surg B Skull Base 77: 96-106, 2016.

21. Kalluri R and Zeisberg M: Fibroblasts in cancer. Nat Rev Cancer 6: 392-401, 2006.

22. Bhowmick NA, Neilson EG and Moses HL: Stromal fibroblasts in cancer initiation and progression. Nature 432: 332-337, 2004.

23. Richards KE, Zeleniak AE, Fishel ML, Wu J, Littlepage LE and Hill R: Cancer-associated fibroblast exosomes regulate survival and proliferation of pancreatic cancer cells. Oncogene 36: 1770-1778, 2017.

24. Li YY, Tao YW, Gao S, Li P, Zheng JM, Zhang SE, Liang J and Zhang Y: Cancer-associated fibroblasts contribute to oral cancer cells proliferation and metastasis via exosome-mediated paracrine miR-34a-5p. EBioMedicine 36: 209-220, 2018.

25. Colotta F, Allavena P, Sica A, Garlanda C and Mantovani A Cancer-related inflammation, the seventh hallmark of cancer: Links to genetic instability. Carcinogenesis 30: 1073-1081, 2009.

26. Voronov E, Shouval DS, Krelin Y, Cagnano E, Benharroch D, Iwakura Y,Dinarello CA and Apte RN: IL-1 is required for tumor invasiveness and angiogenesis. Proc Natl Acad Sci USA 100: 2645-2650, 2003.

27. Apte RN, Dotan S, Elkabets M, White MR, Reich E, Carmi Y, Song X, Dvozkin T, Krelin Y and Voronov E: The involvement of IL-1 in tumorigenesis, tumor invasiveness, metastasis and tumor-host interactions. Cancer Metastasis Rev 25: 387-408, 2006.

28. Chow MT and Luster AD: Chemokines in cancer. Cancer Immunol Res 2: 1125-1131, 2014.

29. He S, He S, Chen CH, Deborde S, Bakst RL, Chernichenko N, McNamara WF, Lee SY, Barajas F, Yu Z, et al: The chemokine (CCL2-CCR2) signaling axis mediates perineural invasion. Mol Cancer Res 13: 380-390, 2015.

30. Muller A, Sonkoly E, Eulert C, Gerber PA, Kubitza R, Schirlau K, Franken-Kunkel P, Poremba C, Snyderman C, Klotz LO, et al: Chemokine receptors in head and neck cancer: Association with metastatic spread and regulation during chemotherapy. Int J Cancer 118: 2147-2157, 2006.

31. Zhu Z, Friess H, diMola FF, Zimmermann A, Graber HU, Korc M and Buchler MW: Nerve growth factor expression correlates with perineural invasion and pain in human pancreatic cancer. J Clin Oncol 17: 2419-2428, 1999.

32. Kolokythas A, Cox DP, Dekker N and Schmidt BL: Nerve growth factor and tyrosine kinase A receptor in oral squamous cell carcinoma: Is there an association with perineural invasion? J Oral Maxillofac Surg 68: 1290-1295, 2010.

33. Zheng P, Chen L, Yuan X, Luo Q, Liu Y, Xie G, Ma Y and Shen L: Exosomal transfer of tumor-associated macrophage-derived miR-21 confers cisplatin resistance in gastric cancer cells. J Exp Clin Cancer Res 36: 53, 2017.

This work is licensed under a Creative Commons Attribution-NonCommercial-NoDerivatives 4.0 International (CC BY-NC-ND 4.0) License. 